\title{
Synthesis of spiro[indolo-1,5-benzodiazepines] from 3-acetyl coumarins for use as possible antianxiety agents
}

\author{
RAVIRAJ A KUSANUR ${ }^{1}$, MANJUNATH GHATE ${ }^{2}$ and MANOHAR V KULKARNI ${ }^{1} *$ \\ ${ }^{1}$ Department of Studies in Chemistry, Karnataka University, Dharwad 580 003, India \\ ${ }^{2}$ Kripanidhi College of Pharmacy, Bangalore, India \\ e-mail: drmvk274@yahoo.co.in
}

MS received 17 May 2004; revised 4 August 2004

\begin{abstract}
Acetyl coumarins (1) when allowed react with isatin (2) gave corresponding 3-(3'-hydroxy-2'-oxo indolo) acetyl coumarins (3), which on dehydration afforded the corresponding $\alpha, \beta$-unsaturated ketones (4). Cyclocondensation of (4) with substituted $o$-phenylene diamines resulted in novel 3-coumarinyl spiro[indolo-1,5-benzodiazepines] (5). Structures of all the compounds have been established on the basis of their IR, NMR and mass spectral data and have been screened for their antimicrobial activity and antianxiety activity in mice.
\end{abstract}

Keywords. Spirobenzodiazepines; distereotopic protons; antianxiety activity.

\section{Introduction}

1,5-Benzodiazepines constitute an important class of psychopharmaca, ${ }^{1}$ in particular as tranquilizers and also as potent virucides and non-nucleoside inhibitors of HIV-1 reverse transcriptase. ${ }^{2}$ Beside this, 1,5-benzodiazepines show antifungal, antibacterial, ${ }^{3}$ antifeedant, ${ }^{4}$ anti-inflammatory, analgesic ${ }^{5}$ and anticonvulsant $^{6}$ activities. The fusion of a heterocyclic system to the benzodiazepine ring appears quite promising for the synthesis of derivatives with greater activity and specificity. Coumarins containing nitrogen heterocycles at $\mathrm{C}_{3}$ position are used as dyes. ${ }^{7}$ They are also used in the manufacture of printed circuits. ${ }^{8}$ Here we report the synthesis of spiro indolo benzodiazepines from 3-acetyl coumarins. The results of the evaluation of antimicrobial and antianxiety activities of these compounds are also reported.

\section{Results and discussion}

3-Acetyl coumarins ${ }^{9,10}$ (1) selectively reacted with the $\mathrm{C}_{3}$-carbonyl of isatin in presence of piperidine to give the $\beta$-hydroxycarbonyl compounds (2). These underwent dehydration under acidic conditions to generate the orange coloured $\alpha, \beta$-unsaturated car-

\footnotetext{
*For correspondence
}

bonyl compounds (4). Cyclo condensation of compounds (4) with o-phenylenediamines resulted in the formation of spiro benzodiazepines (5) (scheme 1).

The IR spectrum of compound $\mathbf{3 a}(\mathrm{R}=\mathrm{H})$ showed three strong bands at 1735,1707 and $1688 \mathrm{~cm}^{-1}$ due

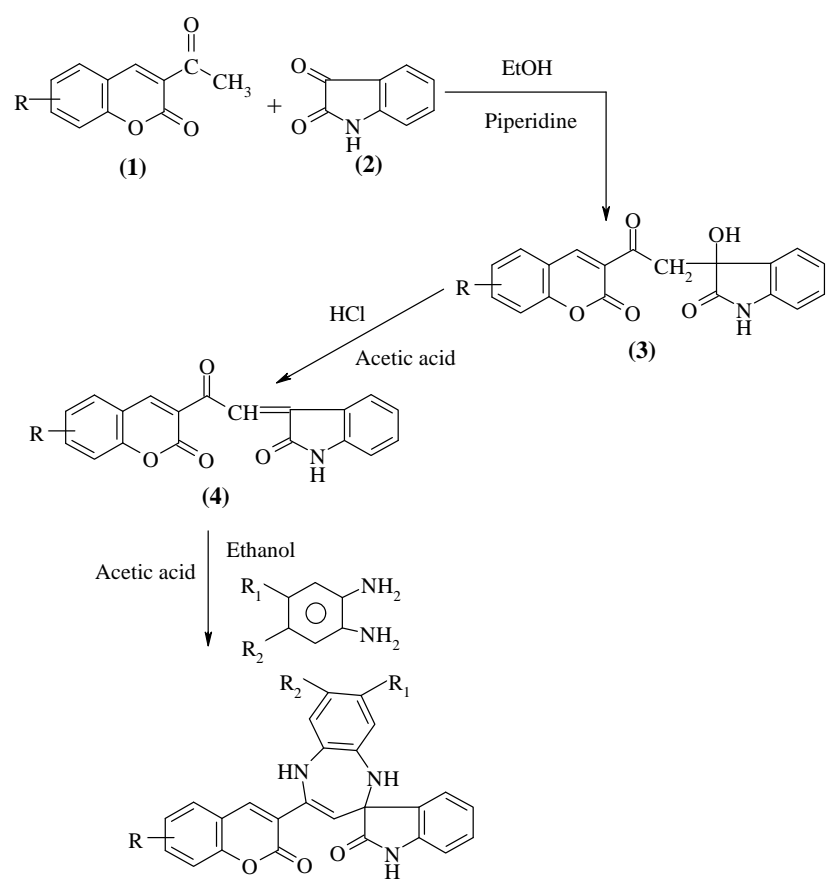

(5)

$\mathrm{R}=\mathrm{H}, 6-\mathrm{CH}_{3}, 8-\mathrm{OCH}_{3}, 5,6$-benzo, 6- $\mathrm{Cl}, 6-\mathrm{Br} ; \mathrm{R}_{1}=\mathrm{R}_{2}=\mathrm{H}, \mathrm{CH}_{3}$

Scheme 1. 
Table 1. IR and PMR spectral data of compounds (3a-f).

\begin{tabular}{|c|c|c|c|c|c|c|c|}
\hline \multirow[b]{2}{*}{ Compd. } & \multirow[b]{2}{*}{$\mathrm{R}$} & \multicolumn{5}{|c|}{$\operatorname{IR}\left(\mathrm{cm}^{-1}\right)$} & \multirow[b]{2}{*}{ PMR $\delta(p p m)$} \\
\hline & & $\mathrm{V}_{\mathrm{C}=\mathrm{O}}$ (lactone) & $V_{\mathrm{C}=\mathrm{O}}$ (keto) & $\mathrm{V}_{\mathrm{C}=\mathrm{O} \text { (amide) }}$ & $v_{\mathrm{NH}}$ & $v_{\mathrm{OH}}$ & \\
\hline $\mathbf{3 a}$ & $\mathrm{H}$ & 1735 & 1707 & 1688 & 3207 & 3491 & $\begin{array}{l}3.58(d, 2 \mathrm{H}, J=17.49 \mathrm{~Hz}), 4 \cdot 13(d, 2 \mathrm{H}, \\
J=17 \cdot 4 \mathrm{~Hz}), 7.63\left(b, s, 1 \mathrm{H}, \mathrm{NH}, \mathrm{D}_{2} \mathrm{O}\right. \\
\text { exchangable }), 3 \cdot 71\left(s, 1 \mathrm{H}, \mathrm{OH}, \mathrm{D}_{2} \mathrm{O}\right. \\
\text { exchangable }), 8 \cdot 53\left(s, 1 \mathrm{H}, \mathrm{C}_{4}-\mathrm{H}\right), \\
6 \cdot 89-7.7(m, 8 \mathrm{H}, \mathrm{Ar}-\mathrm{H})\end{array}$ \\
\hline $\mathbf{3 b}$ & $6-\mathrm{CH}_{3}$ & 1722 & 1701 & 1683 & 3190 & 3404 & $\begin{array}{l}2 \cdot 41\left(s, 3 \mathrm{H}, \mathrm{C}_{6}-\mathrm{CH}_{3}\right), 3 \cdot 59(d, 2 \mathrm{H}, \\
J=17 \cdot 5 \mathrm{~Hz}), 4 \cdot 13(d, 2 \mathrm{H}, J=17 \cdot 6 \mathrm{~Hz}), \\
7 \cdot 7\left(b, s, 1 \mathrm{H}, \mathrm{NH}, \mathrm{D}_{2} \mathrm{O} \text { exchangable }\right), 3 \cdot 73 \\
\left(s, 1 \mathrm{H},-\mathrm{OH}, \mathrm{D}_{2} \mathrm{O} \text { exchangable }\right), 8 \cdot 45 \\
\left(s, 1 \mathrm{H}, \mathrm{C}_{4}-\mathrm{H}\right), 6 \cdot 87-7 \cdot 47(m, 7 \mathrm{H}, \mathrm{Ar}-\mathrm{H})\end{array}$ \\
\hline $3 c$ & $8-\mathrm{OCH}_{3}$ & 1727 & 1705 & 1680 & 3352 & 3409 & $\begin{array}{l}3.96\left(s, 3 \mathrm{H}, 8-\mathrm{OCH}_{3}\right), 3 \cdot 68(d, 2 \mathrm{H}, \\
J=17 \cdot 7 \mathrm{~Hz}), 4 \cdot 07(d, 2 \mathrm{H}, J=17 \cdot 8 \mathrm{~Hz}), \\
10 \cdot 2\left(s, 1 \mathrm{H}, \mathrm{NH}, \mathrm{D}_{2} \mathrm{O} \text { exchangable }\right), \\
3 \cdot 71\left(s, 1 \mathrm{H}, \mathrm{OH}, \mathrm{D}_{2} \mathrm{O} \text { exchangable }\right) \\
8 \cdot 37\left(s, 1 \mathrm{H}, \mathrm{C}_{4}-\mathrm{H}\right), 6 \cdot 81-7 \cdot 32 \\
(m, 7 \mathrm{H}, \mathrm{Ar}-\mathrm{H})\end{array}$ \\
\hline 3d & 5,6-Benzo & 1727 & 1701 & 1672 & 3218 & 3382 & $\begin{array}{l}4 \cdot 22(d, 2 \mathrm{H}, J=17 \cdot 91 \mathrm{~Hz}), 3 \cdot 81(d, 2 \mathrm{H}, \\
J=17 \cdot 8 \mathrm{~Hz}), 9 \cdot 91\left(s, 1 \mathrm{H}, \mathrm{NH}, \mathrm{D}_{2} \mathrm{O}\right. \\
\text { exchangable }), 3 \cdot 67\left(s, 1 \mathrm{H}, \mathrm{OH}, \mathrm{D}_{2} \mathrm{O}\right. \\
\text { exchangable }), 9 \cdot 13\left(s, 1 \mathrm{H}, \mathrm{C}_{4}-\mathrm{H}\right), \\
6 \cdot 88-8 \cdot 29(m, 10 \mathrm{H}, \text { Ar-H })\end{array}$ \\
\hline $3 e$ & $6-\mathrm{Cl}$ & 1725 & 1725 & 1681 & 3192 & 3371 & $\begin{array}{l}4 \cdot 31(d, 2 \mathrm{H}, J=17 \cdot 61 \mathrm{~Hz}), 3 \cdot 72(d, 2 \mathrm{H}, \\
J=17 \cdot 5 \mathrm{~Hz}), 9 \cdot 12\left(s, 1 \mathrm{H}, \mathrm{NH}, \mathrm{D}_{2} \mathrm{O}\right. \\
\text { exchangable }), 3 \cdot 81\left(s, 1 \mathrm{H}, \mathrm{OH}, \mathrm{D}_{2} \mathrm{O}\right. \\
\text { exchangable }), 9 \cdot 12\left(s, 1 \mathrm{H}, \mathrm{C}_{4}-\mathrm{H}\right), \\
6 \cdot 81-7 \cdot 46(m, 7 \mathrm{H}, \mathrm{Ar}-\mathrm{H})\end{array}$ \\
\hline $3 f$ & $6-\mathrm{Br}$ & 1729 & 1730 & 1686 & 3204 & 3381 & $\begin{array}{l}4 \cdot 34(d, 2 \mathrm{H}, J=17 \cdot 84 \mathrm{~Hz}), 3 \cdot 81(d, 2 \mathrm{H}, \\
J=17 \cdot 7 \mathrm{~Hz}), 8 \cdot 84\left(s, 1 \mathrm{H}, \mathrm{NH}, \mathrm{D}_{2} \mathrm{O}\right. \\
\text { exchangable }), 3 \cdot 74\left(s, 1 \mathrm{H}, \mathrm{OH}, \mathrm{D}_{2} \mathrm{O}\right. \\
\text { exchangable }), 9 \cdot 14\left(s, 1 \mathrm{H}, \mathrm{C}_{4}-\mathrm{H}\right), \\
6 \cdot 79-7 \cdot 48(m, 7 \mathrm{H}, \mathrm{Ar}-\mathrm{H})\end{array}$ \\
\hline
\end{tabular}

to lactone, ketone and amide carbonyls respectively. In the PMR spectrum of $\mathbf{3 a}(\mathrm{R}=\mathrm{H})$, the diastereotopic $\mathrm{CH}_{2}$ protons appeared as two separate doublets at 3.50 and $4.13 \mathrm{ppm}(J=17.5 \mathrm{~Hz})$. The $\mathrm{NH}$ and $\mathrm{OH}$ protons were characterized by $\mathrm{D}_{2} \mathrm{O}$ exchange. The other protons resonated at expected fields (table 1).

The $\beta$-hydroxy ketones (3) readily underwent dehydration in presence of an acid to yield $\alpha, \beta$-unsaturated ketones (4). The formation of enones was characterized by the absence of $\mathrm{OH}$ stretching and the shift of keto carbonyl stretching to lower frequency at $\sim 1620 \mathrm{~cm}^{-1}$ in their IR spectra. The IR spectrum of compound $4 \mathbf{a}(\mathrm{R}=\mathrm{H})$ shows band at $1721 \mathrm{~cm}^{-1}$ due to $v_{\mathrm{C}=\mathrm{O}}$ of lactone and bands at 1610 and $1622 \mathrm{~cm}^{-1}$ due to $v_{\mathrm{C}=\mathrm{O}}$ of keto and $\mathrm{v}_{\mathrm{C}=\mathrm{O}}$ of amide respectively. The PMR spectrum of compound $4 \mathbf{a}$ $(\mathrm{R}=\mathrm{H})$ showed a singlet at $7.93 \mathrm{ppm}$ due to olefinic proton and singlet at $8.70 \mathrm{ppm}$ due to $\mathrm{C}_{4}-\mathrm{H}$ of coumarin. Aromatic protons resonated in the region 6.96-7.83 ppm. The $\mathrm{NH}$ proton was observed at 10.46 ppm, which was confirmed by $\mathrm{D}_{2} \mathrm{O}$ exchange (table 2).

$\alpha, \beta$-Unsaturated ketones (4) when treated with $o$ phenylene diamine in ethanol afforded spirobenzodiazepines (5). In the IR spectrum of compound $\mathbf{5 a}$ ( $\mathrm{R}=\mathrm{H}, \mathrm{R}_{1}=\mathrm{R}_{2}=\mathrm{H}$ ) the $\mathrm{N}-\mathrm{H}$ stretching band observed at $3435 \mathrm{~cm}^{-1}$ (table 3 ). The absence of ketocarbonyl stretching confirms the conversion of $\alpha, \beta$ unsaturated ketones into spirobenzodiazepines (5). Lactone and amide carbonyls appeared at 1716 and $1673 \mathrm{~cm}^{-1}$ respectively. The PMR spectrum of com- 
Table 2. IR and PMR spectral data of compounds $4(\mathbf{a}-\mathbf{f})$.

\begin{tabular}{|c|c|c|c|c|c|c|}
\hline \multirow[b]{2}{*}{ Compd. } & \multirow[b]{2}{*}{$\mathrm{R}$} & \multicolumn{4}{|c|}{$\operatorname{IR}\left(\mathrm{cm}^{-1}\right)$} & \multirow[b]{2}{*}{$\operatorname{PMR} \delta(\mathrm{ppm})$} \\
\hline & & $\mathrm{V}_{\mathrm{C}=\mathrm{O}}$ (lactone) & $\mathrm{V}_{\mathrm{C}=\mathrm{O} \text { (keto) }}$ & $\mathrm{V}_{\mathrm{C}=\mathrm{O} \text { (amide) }}$ & $v_{\mathrm{N}-\mathrm{H}}$ & \\
\hline $4 a$ & $\mathrm{H}$ & 1721 & 1610 & 1662 & 3188 & $\begin{array}{l}7.93(s, 1 \mathrm{H},=\mathrm{CH}), 8 \cdot 70\left(s, 1 \mathrm{H}, \mathrm{C}_{4}-\mathrm{H}\right), 10 \cdot 46 \\
\left(s, 1 \mathrm{H}, \mathrm{NH}, \mathrm{D}_{2} \mathrm{O} \text { exchangable }\right), 7 \cdot 83\left(d, 1 \mathrm{H}, \mathrm{C}_{5}-\mathrm{H},\right. \\
J=7 \cdot 48 \mathrm{~Hz}), 7 \cdot 73\left(t, 1 \mathrm{H}, \mathrm{C}_{6}-\mathrm{H}, J=8 \cdot 3 \mathrm{~Hz}\right), 7 \cdot 40 \\
\left(t, \mathrm{C}_{7}-\mathrm{H}, J=8 \cdot 1 \mathrm{~Hz}\right), 8 \cdot 45\left(d, 1 \mathrm{H}, \mathrm{C}_{8}-\mathrm{H}, 7 \cdot 74 \mathrm{~Hz}\right), \\
6 \cdot 87\left(d, 1 \mathrm{H}, \mathrm{C}_{4^{\prime}}-\mathrm{H}, J=7 \cdot 8 \mathrm{~Hz}\right), 6 \cdot 96\left(t, 1 \mathrm{H}, \mathrm{C}_{5}^{\prime}-\mathrm{H},\right. \\
J=7 \cdot 34 \mathrm{~Hz}), 7 \cdot 40\left(t, 1 \mathrm{H}, \mathrm{C}_{6}{ }^{\prime}-\mathrm{H}, J=7 \cdot 21\right), 7 \cdot 42 \\
\left(d, 1 \mathrm{H}, \mathrm{C}_{7}{ }^{\prime}-\mathrm{H}, J=7 \cdot 2 \mathrm{~Hz}\right)\end{array}$ \\
\hline $4 b$ & $6-\mathrm{CH}_{3}$ & 1727 & 1622 & 1661 & 3196 & $\begin{array}{l}2.46\left(s, 3 \mathrm{H}, \mathrm{C}_{6}-\mathrm{CH}_{3}\right), 7 \cdot 94(s, 1 \mathrm{H},=\mathrm{CH}), 8 \cdot 59(s, \\
\left.1 \mathrm{H}, \mathrm{C}_{4}-\mathrm{H}\right), 10 \cdot 33\left(s, 1 \mathrm{H}, \mathrm{NH}, \mathrm{D}_{2} \mathrm{O} \text { exchangable }\right), \\
7 \cdot 57\left(s, 1 \mathrm{H}, \mathrm{C}_{5}-\mathrm{H}\right), 7.52\left(d, 1 \mathrm{H}, \mathrm{C}_{7}-\mathrm{H}, J=7 \cdot 8 \mathrm{~Hz}\right), \\
8.46\left(d, 1 \mathrm{H}, \mathrm{C}_{8}-\mathrm{H}, J=7 \cdot 66 \mathrm{~Hz}\right), 6 \cdot 86(d, 1 \mathrm{H}, \\
\left.\mathrm{C}_{4}^{\prime}-\mathrm{H}, J=7.6 \mathrm{~Hz}\right), 6.37\left(t, 1, \mathrm{C}_{5}^{\prime}-\mathrm{H}, J=7.6 \mathrm{~Hz}\right), \\
7 \cdot 31\left(t, 1 \mathrm{H}, \mathrm{C}_{6}^{\prime}-\mathrm{H}, J=7.7 \mathrm{~Hz}\right), 7 \cdot 53\left(d, 1 \mathrm{H}, \mathrm{C}_{7}^{\prime}-\mathrm{H},\right. \\
J=7 \cdot 6 \mathrm{~Hz})\end{array}$ \\
\hline $4 c$ & $8-\mathrm{OCH}_{3}$ & 1733 & 1606 & 1655 & 3306 & $\begin{array}{l}4.00\left(s, 3 \mathrm{H}, 8-\mathrm{OCH}_{3}\right), 7.93(s, 1 \mathrm{H},=\mathrm{CH}), 8.64 \\
\left(\mathrm{~s}, 1 \mathrm{H}, \mathrm{C}_{4}-\mathrm{H}\right), 10 \cdot 40\left(1 \mathrm{H}, \mathrm{NH}, \mathrm{D}_{2} \mathrm{O} \text { exchangable }\right) \\
6.85-8.47(m, 7 \mathrm{H}, \mathrm{Ar}-\mathrm{H})\end{array}$ \\
\hline $4 d$ & 5,6-Benzo & 1727 & 1601 & 1645 & 3186 & $\begin{array}{l}10 \cdot 56\left(s, 1 \mathrm{H}, \mathrm{D}_{2} \mathrm{O} \text { exchangable }\right), 9.46(s, 1 \mathrm{H}, \\
\left.\mathrm{C}_{4}-\mathrm{H}\right), 7 \cdot 90(s, 1 \mathrm{H},=\mathrm{CH}), 6 \cdot 87-8.47(m, 7 \mathrm{H}, \\
\text { Ar-H })\end{array}$ \\
\hline $4 e$ & $6-\mathrm{Cl}$ & 1724 & 1628 & 1676 & 3371 & $\begin{array}{l}7.96(s, 1 \mathrm{H},=\mathrm{CH}), 8 \cdot 71\left(s, 1 \mathrm{H}, \mathrm{C}_{4}-\mathrm{H}\right), 10 \cdot 50(1 \mathrm{H}, \\
\left.\mathrm{NH}, \mathrm{D}_{2} \mathrm{O} \text { exchangable }\right), 6 \cdot 47-7.96(m, 7 \mathrm{H}, \text { Ar-H })\end{array}$ \\
\hline $4 f$ & $6-\mathrm{Br}$ & 1729 & 1641 & 1669 & 3324 & $\begin{array}{l}7 \cdot 88(s, 1 \mathrm{H},=\mathrm{CH}), 7 \cdot 69\left(s, 1 \mathrm{H}, \mathrm{C}_{4}-\mathrm{H}\right), 10 \cdot 46 \\
\left(1 \mathrm{H}, \mathrm{NH}, \mathrm{D}_{2} \mathrm{O} \text { exchangable }\right), 6 \cdot 5-8 \cdot 10(m, 7 \mathrm{H}, \\
\text { Ar-H) }\end{array}$ \\
\hline
\end{tabular}

pound 5a $\left(\mathrm{R}=\mathrm{H}, \mathrm{R}_{1}=\mathrm{R}_{2}=\mathrm{H}\right)$ shows a singlet at $8.87 \mathrm{ppm}$ due to $\mathrm{C}_{4}-\mathrm{H}$ of coumarin (table 3 ). The olefinic proton of benzodiaepine resonated as singlet at $8.93 \mathrm{ppm}$, whereas aromatic protons resonated as multiplet at $7 \cdot 28-8 \cdot 16 \mathrm{ppm}$.

EI mass spectrum of compound 5a $(\mathrm{R}=\mathrm{H}$, $\left.\mathrm{R}_{1}=\mathrm{R}_{2}=\mathrm{H}\right) \mathrm{m} / \mathrm{z} 407\left(M^{+} 5 \%\right), 274(100 \%), 246$ (35\%), $219(11 \%), 190(10 \%), 172(8 \%), 69(30 \%)$ confirmed the formation of benzodiazepins.

\section{Biological activity}

\subsection{Antimicrobial activity}

Antimicrobial activity was carried out against two pathogenic bacteria E. coli, and B. subtillis, and A. niger as the fungal strain. The reference drugs used were ciprofloxacin and griseofulvin respectively. The tests were carried out by cup plate method. ${ }^{11}$ Among all the benzodiazepino coumarins 5, 5f with $\mathrm{R}=8-\mathrm{OCH}_{3} . \mathrm{R}_{1}=\mathrm{R}_{2}=\mathrm{CH}_{3}$ show $88.88 \%$ inhibition against $B$. substillis and $77.77 \%$ of inhibition against E. coli as compared to the standard and other compounds are moderately active. Compounds $\mathbf{5 b}$ and $\mathbf{5 i}$ show $77.77 \%$ of inhibition against $A$. niger and other compounds are moderately active. The results are shown in table 4.

\subsection{Antianxiety activity in mice}

All the newly synthesised benzodiazepines were screened for their antianxiety activity in mice on plus maze apparatus devised by Crawley and Godwin, ${ }^{12}$ modified by $\mathrm{T}$ Kilofoil ${ }^{13}$ using sodiumpentabarbitone as the standard.

The apparatus consists of plexiglass box $(40 \times$ $21 \times 21$ inches) divided into two chambers by black plexiglass partition. The box was placed within a layer of soundproof box, which is equipped with one-way observation window. The partition dividing the two chambers $13 \times 5$ inch opening, through which animal could easily pass. The dark chamber 
Table 3. IR and PMR spectral data of compounds 5(a-l).

\begin{tabular}{|c|c|c|c|c|c|c|c|}
\hline \multirow[b]{2}{*}{ Compd. } & \multirow[b]{2}{*}{$\mathrm{R}$} & \multirow[b]{2}{*}{$\mathrm{R}_{1}$} & \multirow[b]{2}{*}{$\mathrm{R}_{2}$} & \multicolumn{3}{|c|}{$\operatorname{IR}\left(\mathrm{cm}^{-1}\right)$} & \multirow[b]{2}{*}{ PMR $\delta(p p m)$} \\
\hline & & & & $\mathrm{V}_{\mathrm{C}=\mathrm{O} \text { (lactone) }}$ & $\mathrm{V}_{\mathrm{C}=\mathrm{O} \text { (amide) }}$ & $\mathrm{v}_{\mathrm{N}-\mathrm{H}}$ & \\
\hline $5 \mathbf{a}$ & $\mathrm{H}$ & $\mathrm{H}$ & $\mathrm{H}$ & 1716 & 1673 & 3435 & $\begin{array}{l}8 \cdot 87\left(s, 1 \mathrm{H}, \mathrm{C}_{4}-\mathrm{H}\right), 9 \cdot 83(s, 1 \mathrm{H},=\mathrm{CH}) \\
7 \cdot 28-8 \cdot 16(m, 12 \mathrm{H}, \mathrm{Ar}-\mathrm{H})\end{array}$ \\
\hline $5 \mathbf{b}$ & $\mathrm{H}$ & $\mathrm{CH}_{3}$ & $\mathrm{CH}_{3}$ & 1701 & 1632 & 3272 & $\begin{array}{l}2.52\left(s, 6 \mathrm{H},-\mathrm{CH}_{3}\right), 8 \cdot 80\left(s, 1 \mathrm{H}, \mathrm{C}_{4}-\mathrm{H}\right), 9 \cdot 71 \\
(s, 1 \mathrm{H},=\mathrm{CH}), 7 \cdot 26-7 \cdot 89(m, 10 \mathrm{H}, \mathrm{Ar}-\mathrm{H})\end{array}$ \\
\hline $5 c$ & $6-\mathrm{CH}_{3}$ & $\mathrm{H}$ & $\mathrm{H}$ & 1705 & 1629 & 3399 & $\begin{array}{l}2.45\left(s, 3 \mathrm{H}, \mathrm{C}_{6}-\mathrm{CH}_{3}\right), 8 \cdot 78\left(s, 1 \mathrm{H}, \mathrm{C}_{4}-\mathrm{H}\right) \\
9.80(s, 1 \mathrm{H},=\mathrm{CH}), 7 \cdot 26-7.90(m, 11 \mathrm{H}, \mathrm{Ar}-\mathrm{H})\end{array}$ \\
\hline $5 d$ & $6-\mathrm{CH}_{3}$ & $\mathrm{CH}_{3}$ & $\mathrm{CH}_{3}$ & 1716 & 1671 & 3404 & $\begin{array}{l}2 \cdot 45\left(s, 3 \mathrm{H}, \mathrm{C}_{6}-\mathrm{CH}_{3}\right), 2 \cdot 52\left(s, 6 \mathrm{H},-\mathrm{CH}_{3}\right) \\
8 \cdot 75\left(s, 1 \mathrm{H}, \mathrm{C}_{4}-\mathrm{H}\right), 9 \cdot 70(s, 1 \mathrm{H},=\mathrm{CH}) \\
7 \cdot 26-7.91(m, 9 \mathrm{H}, \mathrm{Ar}-\mathrm{H})\end{array}$ \\
\hline $5 e$ & $8-\mathrm{OCH}_{3}$ & $\mathrm{H}$ & $\mathrm{H}$ & 1712 & 1656 & 3427 & $\begin{array}{l}4.02\left(s, 3 \mathrm{H}, 6-\mathrm{OCH}_{3}\right), 8 \cdot 84\left(s, 1 \mathrm{H}, \mathrm{C}_{4}-\mathrm{H}\right), \\
9 \cdot 84(s, 1 \mathrm{H},=\mathrm{CH}), 7 \cdot 15-8 \cdot 19(m, 11 \mathrm{H}, \mathrm{Ar}-\mathrm{H})\end{array}$ \\
\hline $5 f$ & $8-\mathrm{OCH}_{3}$ & $\mathrm{CH}_{3}$ & $\mathrm{CH}_{3}$ & 1722 & 1694 & 3399 & $\begin{array}{l}2 \cdot 53\left(s, 6 \mathrm{H}, \mathrm{CH}, \mathrm{CH}_{3}\right), 4 \cdot 02(s, 3 \mathrm{H} \\
\left.\mathrm{C}_{6}-\mathrm{OCH}_{3}\right), 8 \cdot 80\left(s, 1 \mathrm{H}, \mathrm{C}_{4}-\mathrm{H}\right), 9 \cdot 74(s, 1 \mathrm{H}, \\
=\mathrm{CH}), 7 \cdot 14-7.94(m, 9 \mathrm{H}, \mathrm{Ar}-\mathrm{H})\end{array}$ \\
\hline $5 g$ & 5,6-Benzo & $\mathrm{H}$ & $\mathrm{H}$ & 1716 & 1651 & 3420 & $\begin{array}{l}9 \cdot 68\left(s, 14 \mathrm{H}, \mathrm{C}_{4}-\mathrm{H}\right), 9 \cdot 84(s, 1 \mathrm{H},=\mathrm{CH}), \\
6 \cdot 92-8 \cdot 58(m, 14 \mathrm{H}, \text { Ar-H })\end{array}$ \\
\hline $5 h$ & 5,6-Benzo & $\mathrm{CH}_{3}$ & $\mathrm{CH}_{3}$ & 1716 & 1662 & 3404 & $\begin{array}{l}2.56\left(s, 6 \mathrm{H}, \mathrm{CH}_{3}\right), 9 \cdot 32\left(s, 1 \mathrm{H}, \mathrm{C}_{4}-\mathrm{H}\right), 9 \cdot 80 \\
(s, 1 \mathrm{H},=\mathrm{CH}), 6 \cdot 92-8.58(m, 12 \mathrm{H}, \mathrm{Ar}-\mathrm{H})\end{array}$ \\
\hline $5 \mathbf{i}$ & $6-\mathrm{Cl}$ & $\mathrm{H}$ & $\mathrm{H}$ & 1712 & 1684 & 3394 & $\begin{array}{l}8 \cdot 91\left(s, 1 \mathrm{H} \mathrm{C}_{4}-\mathrm{H}\right), 9 \cdot 83(s, 1 \mathrm{H},=\mathrm{CH}) \\
7 \cdot 29-8 \cdot 2(m, 11 \mathrm{H}, \mathrm{Ar}-\mathrm{H})\end{array}$ \\
\hline $5 \mathbf{j}$ & $6-\mathrm{Cl}$ & $\mathrm{CH}_{3}$ & $\mathrm{CH}_{3}$ & 1713 & 1640 & 3328 & $\begin{array}{l}2.54\left(s, 6 \mathrm{H}, \mathrm{CH}_{3}\right), 8.92\left(s, 1 \mathrm{H}, \mathrm{C}_{4}-\mathrm{H}\right), 9.86 \\
(s, 1 \mathrm{H},=\mathrm{CH}), 7 \cdot 34-8.4(m, 9 \mathrm{H}, \mathrm{Ar}-\mathrm{H})\end{array}$ \\
\hline $5 \mathbf{k}$ & $6-\mathrm{Br}$ & $\mathrm{H}$ & $\mathrm{H}$ & 1704 & 1639 & 3298 & $\begin{array}{l}8.94\left(s, 1 \mathrm{H}, \mathrm{C}_{4}-\mathrm{H}\right), 9 \cdot 84(s, 1 \mathrm{H},=\mathrm{CH}) \\
7 \cdot 29-8.40(m, 11 \mathrm{H}, \mathrm{Ar}-\mathrm{H})\end{array}$ \\
\hline 51 & $6-\mathrm{Br}$ & $\mathrm{CH}_{3}$ & $\mathrm{CH}_{3}$ & 1708 & 1646 & 3204 & $\begin{array}{l}2.56\left(s, 6 \mathrm{H}, \mathrm{CH}_{3}\right), 8 \cdot 86\left(s, 1 \mathrm{H}, \mathrm{C}_{6}-\mathrm{H}\right), 9.86 \\
(s, 1 \mathrm{H},=\mathrm{CH}), 7 \cdot 34-8.4(m, 9 \mathrm{H}, \mathrm{Ar}-\mathrm{H})\end{array}$ \\
\hline
\end{tabular}

$(14 \times 21 \times 21$ inch $)$ was made up of dark plexiglass except for the side ferry observation window. This side is clear and covered with black plastic. The testing was performed between 12.00 noon to 6.00 p.m. in an isolated darkened laboratory.

Mice weighing $25 \mathrm{~g}$ were chosen and were sorted into five animals in a group. They were allowed free access to food, water and libitum. Animals were given $60 \mathrm{~min}$ time to acclimatize to the environment prior to the administration of drugs. Drugs or test samples in DMF were given at the dose of $20 \mathrm{mg} / \mathrm{kg}$ body weight from here each animal was individually placed in the centre of the light area of the apparatus and observed for $10 \mathrm{~min}$. The total amount of time spent in dark area is measured. Lesser the time spent in dark space the greater is the antianxiety activity of the drugs. The results are given in table 5. Compound 5a with $\mathrm{R}=\mathrm{H}, \mathrm{R}_{1}=\mathrm{R}_{2}=\mathrm{H}$ have shown comparable activity with the standard and the other compounds are moderately active.

\section{Experimental}

Melting points were taken in open capillaries and are uncorrected. Purity of the compounds was checked by TLC. IR spectra were recorded in $\mathrm{KBr}$ on Perkin-Elmer spectrophotometer $\left(V\right.$ in $\left.\mathrm{cm}^{-1}\right)$ and PMR spectra on Bruker-300 MHz FTNMR spectrometer using TMS as internal standard (chemical shifts in $\delta$, ppm). Mass spectra were recorded on a Jeol JMS D-300 instrument at $70 \mathrm{ev.}$

\section{$4.13-\left(3^{\prime}\right.$-hydroxy-2'-oxo indolo $)$ acetyl coumarins 3(a-e)}

A mixture of 3-acetyl coumarin (1.88 g, 0.01 mole), isatin $(1.47 \mathrm{~g}, 0.01$ mole) in absolute alcohol (100 $\mathrm{ml})$ and two drops of piperidine was stirred for half an hour at room temperature. The reaction mixture was allowed to stand for overnight at room temperature. The solid separated was filtered and 
Table 4. Antimicrobial activity of compounds $\mathbf{5}(\mathbf{a}-\mathbf{l})$.

\begin{tabular}{|c|c|c|c|c|c|c|c|c|c|}
\hline \multirow[b]{2}{*}{ Compd. } & \multirow[b]{2}{*}{$\mathrm{R}$} & \multirow[b]{2}{*}{$\mathrm{R}_{1}$} & \multirow[b]{2}{*}{$\mathrm{R}_{2}$} & \multicolumn{2}{|c|}{ B. subtillis } & \multicolumn{2}{|c|}{ E. coli } & \multicolumn{2}{|c|}{ A. niger } \\
\hline & & & & $\begin{array}{c}\text { Zone of } \\
\text { inhibition } \\
(\mathrm{mm})\end{array}$ & $\begin{array}{c}\text { Relative } \\
\text { inhibition } \\
(\%)\end{array}$ & $\begin{array}{c}\text { Zone of } \\
\text { inhibition } \\
(\mathrm{mm})\end{array}$ & $\begin{array}{c}\text { Relative } \\
\text { inhibition } \\
(\%)\end{array}$ & $\begin{array}{c}\text { Zone of } \\
\text { inhibition } \\
(\mathrm{mm})\end{array}$ & $\begin{array}{c}\text { Relative } \\
\text { inhibition } \\
\quad(\%)\end{array}$ \\
\hline $5 \mathbf{a}$ & $\mathrm{H}$ & $\mathrm{H}$ & $\mathrm{H}$ & 18 & $66 \cdot 66$ & 17 & $61 \cdot 11$ & 15 & $50 \cdot 00$ \\
\hline $5 \mathbf{b}$ & $\mathrm{H}$ & $\mathrm{CH}_{3}$ & $\mathrm{CH}_{3}$ & 16 & $55 \cdot 55$ & 17 & $61 \cdot 11$ & 20 & $77 \cdot 77$ \\
\hline $5 c$ & 6- $\mathrm{CH}_{3}$ & $\mathrm{H}$ & $\mathrm{H}$ & 12 & $33 \cdot 33$ & 10 & $22 \cdot 22$ & 12 & $33 \cdot 33$ \\
\hline $5 d$ & 6- $\mathrm{CH}_{3}$ & $\mathrm{CH}_{3}$ & $\mathrm{CH}_{3}$ & 10 & $22 \cdot 22$ & 10 & $22 \cdot 22$ & 10 & $22 \cdot 22$ \\
\hline $5 e$ & $8-\mathrm{OCH}_{3}$ & $\mathrm{H}$ & $\mathrm{H}$ & 18 & $66 \cdot 66$ & 17 & $61 \cdot 11$ & 18 & $66 \cdot 66$ \\
\hline $5 f$ & $8-\mathrm{OCH}_{3}$ & $\mathrm{CH}_{3}$ & $\mathrm{CH}_{3}$ & 22 & $88 \cdot 88$ & 20 & $77 \cdot 77$ & 20 & $77 \cdot 77$ \\
\hline $5 g$ & 5,6-Benzo & $\mathrm{H}$ & $\mathrm{H}$ & 18 & $61 \cdot 11$ & 16 & $55 \cdot 55$ & 15 & $50 \cdot 00$ \\
\hline $5 h$ & 5,6-Benzo & $\mathrm{CH}_{3}$ & $\mathrm{CH}_{3}$ & 18 & $61 \cdot 11$ & 10 & $22 \cdot 22$ & 12 & $33 \cdot 33$ \\
\hline $5 \mathbf{i}$ & $6-\mathrm{Cl}$ & $\mathrm{H}$ & $\mathrm{H}$ & 18 & $66 \cdot 66$ & 19 & $72 \cdot 22$ & 20 & $77 \cdot 77$ \\
\hline $5 \mathbf{j}$ & 6-Cl & $\mathrm{CH}_{3}$ & $\mathrm{CH}_{3}$ & 17 & $61 \cdot 11$ & 16 & $55 \cdot 55$ & 14 & $44 \cdot 44$ \\
\hline $5 \mathbf{k}$ & $6-\mathrm{Br}$ & $\mathrm{H}$ & $\mathrm{H}$ & 18 & $66 \cdot 66$ & 19 & $72 \cdot 22$ & 16 & $55 \cdot 55$ \\
\hline 51 & $6-\mathrm{Br}$ & $\mathrm{CH}_{3}$ & $\mathrm{CH}_{3}$ & 17 & $61 \cdot 11$ & 16 & $55 \cdot 55$ & 16 & $55 \cdot 55$ \\
\hline DMF & & & & 06 & - & 06 & - & 06 & - \\
\hline Ciprofloxacin & & & & 24 & 100 & 24 & 100 & - & - \\
\hline Griseofulvin & & & & - & - & - & - & 24 & 100 \\
\hline
\end{tabular}

Table 5. Results of antianxiety activity in mice.

\begin{tabular}{llccc}
\hline Compd. & \multicolumn{1}{c}{$\mathrm{R}$} & $\mathrm{R}_{1}$ & $\mathrm{R}_{2}$ & $\begin{array}{c}\text { Time spent in } \\
\text { dark space (s) }\end{array}$ \\
\hline $\mathbf{5 a}$ & $\mathrm{H}$ & $\mathrm{H}$ & $\mathrm{H}$ & $308 \pm 12 \cdot 0$ \\
$\mathbf{5 b}$ & $\mathrm{H}$ & $\mathrm{CH}_{3}$ & $\mathrm{CH}_{3}$ & $320 \pm 16 \cdot 8$ \\
$\mathbf{5 c}$ & $6-\mathrm{CH}_{3}$ & $\mathrm{H}$ & $\mathrm{H}$ & $318 \pm 18 \cdot 5$ \\
$\mathbf{5 d}$ & $6-\mathrm{CH}_{3}$ & $\mathrm{CH}_{3}$ & $\mathrm{CH}_{3}$ & $358 \pm 15 \cdot 0$ \\
$\mathbf{5 e}$ & $8-\mathrm{OCH}_{3}$ & $\mathrm{H}$ & $\mathrm{H}$ & $314 \pm 12 \cdot 0$ \\
$\mathbf{5 f}$ & $8-\mathrm{OCH}_{3}$ & $\mathrm{CH}_{3}$ & $\mathrm{CH}_{3}$ & $315 \pm 09 \cdot 8$ \\
$\mathbf{5 g}$ & $5,6-\mathrm{Benzo}$ & $\mathrm{H}$ & $\mathrm{H}$ & $316 \pm 08 \cdot 9$ \\
$\mathbf{5 h}$ & $5,6-\mathrm{Benzo}$ & $\mathrm{CH}_{3}$ & $\mathrm{CH}_{3}$ & $319 \pm 11 \cdot 1$ \\
$\mathbf{5 i}$ & $6-\mathrm{Cl}$ & $\mathrm{H}$ & $\mathrm{H}$ & $324 \pm 12 \cdot 1$ \\
$\mathbf{5 j}$ & $6-\mathrm{Cl}$ & $\mathrm{CH}_{3}$ & $\mathrm{CH}_{3}$ & $328 \pm 12 \cdot 1$ \\
$\mathbf{5 k}$ & $6-\mathrm{Br}$ & $\mathrm{H}$ & $\mathrm{H}$ & $318 \pm 09 \cdot 4$ \\
$\mathbf{5 l}$ & $6-\mathrm{Br}$ & $\mathrm{CH}_{3}$ & $\mathrm{CH}_{3}$ & $321 \pm 08 \cdot 4$ \\
Control & & & & $401 \cdot 3 \pm 25 \cdot 0$ \\
Pentabarbitone & & & $295 \pm 11 \cdot 51$ \\
\hline
\end{tabular}

recrystallised from alcohol-dioxane mixture to afford the hydroxy compound $\mathbf{3 a}(3 \cdot 12 \mathrm{~g}, 93 \cdot 13 \%)$.

\subsection{3 -[2'-oxo-3'-indolo] $\alpha, \beta$-unsaturated ketones $4(\mathbf{a}-\mathbf{e})$}

To the solution of 3-(3'-hydroxy-2'-oxo indolo) acetyl coumarin (3a) (3.35 g, 0.01 mole) in acetic acid $(25 \mathrm{ml}), 0.5 \mathrm{ml}$ of concentrated hydrochloric acid was added. The reaction mixture was warmed on water bath for one hour and cooled to room temperature. The separated orange coloured precipitate was filtered and recrystallised from alcohol dioxane mixture to afford the ketone $\mathbf{4 a}(2.90 \mathrm{~g}, 91.48 \%)$.

\section{$4.344^{\prime}$-(coumarin-3-yl)spiro[3H-indole-3,2'-1,5- benzodiazepine ]-2(1H)-one}

To a solution of 3-[(2'-oxo-3'-indolo $\alpha, \beta$-unsaturated ketones (4a) (1.58 g 0.005 mole) in ethanol 
Table 6. Physical and analytical data of compounds 5(a-l).

\begin{tabular}{|c|c|c|c|c|c|c|c|c|c|c|}
\hline \multirow[b]{2}{*}{ Compd. } & \multirow[b]{2}{*}{$\mathrm{R}$} & \multirow[b]{2}{*}{$\mathrm{R}_{1}$} & \multirow[b]{2}{*}{$\mathrm{R}_{2}$} & \multirow[b]{2}{*}{ Solvent } & \multirow{2}{*}{$\begin{array}{l}\text { MP } \\
\left({ }^{\circ} \mathrm{C}\right)\end{array}$} & \multirow{2}{*}{$\begin{array}{l}\text { Yield } \\
(\%)\end{array}$} & \multirow{2}{*}{$\begin{array}{l}\text { Mol. } \\
\text { formula }\end{array}$} & \multicolumn{3}{|c|}{ Analysis found (calc.) (\%) } \\
\hline & & & & & & & & $\mathrm{C}$ & $\mathrm{H}$ & $\mathrm{N}$ \\
\hline $5 \mathbf{a}$ & $\mathrm{H}$ & $\mathrm{H}$ & $\mathrm{H}$ & Ethanol & 183 & $70 \cdot 88$ & $\mathrm{C}_{25} \mathrm{H}_{17} \mathrm{~N}_{3} \mathrm{O}_{3}$ & $\begin{array}{c}73 \cdot 68 \\
(73 \cdot 71)\end{array}$ & $\begin{array}{l}4 \cdot 15 \\
(4 \cdot 17)\end{array}$ & $\begin{array}{l}10 \cdot 2 \\
(10 \cdot 31)\end{array}$ \\
\hline $5 \mathbf{b}$ & $\mathrm{H}$ & $\mathrm{CH}_{3}$ & $\mathrm{CH}_{3}$ & Ethanol & 178 & $69 \cdot 11$ & $\mathrm{C}_{27} \mathrm{H}_{21} \mathrm{~N}_{3} \mathrm{O}_{3}$ & $\begin{array}{c}74 \cdot 46 \\
(74 \cdot 48)\end{array}$ & $\begin{array}{c}4 \cdot 79 \\
(4 \cdot 82)\end{array}$ & $\begin{array}{c}9 \cdot 63 \\
(9 \cdot 65)\end{array}$ \\
\hline $5 c$ & $6-\mathrm{CH}_{3}$ & $\mathrm{H}$ & $\mathrm{H}$ & Ethanol & 171 & $74 \cdot 12$ & $\mathrm{C}_{26} \mathrm{H}_{19} \mathrm{~N}_{3} \mathrm{O}_{3}$ & $\begin{array}{c}74 \cdot 05 \\
(74 \cdot 10)\end{array}$ & $\begin{array}{c}4 \cdot 48 \\
(4 \cdot 51)\end{array}$ & $\begin{array}{c}9.94 \\
(9.97)\end{array}$ \\
\hline $5 d$ & $6-\mathrm{CH}_{3}$ & $\mathrm{CH}_{3}$ & $\mathrm{CH}_{3}$ & Ethanol & 215 & $72 \cdot 30$ & $\mathrm{C}_{28} \mathrm{H}_{23} \mathrm{~N}_{3} \mathrm{O}_{4}$ & $\begin{array}{c}74 \cdot 81 \\
(74 \cdot 85)\end{array}$ & $\begin{array}{l}5 \cdot 09 \\
(5 \cdot 12)\end{array}$ & $\begin{array}{c}9 \cdot 31 \\
(9 \cdot 35)\end{array}$ \\
\hline $5 e$ & $8-\mathrm{OCH}_{3}$ & $\mathrm{H}$ & $\mathrm{H}$ & Ethanol & 208 & $65 \cdot 81$ & $\mathrm{C}_{26} \mathrm{H}_{19} \mathrm{~N}_{3} \mathrm{O}_{4}$ & $\begin{array}{l}71 \cdot 36 \\
(71 \cdot 39)\end{array}$ & $\begin{array}{c}4 \cdot 31 \\
(4 \cdot 34)\end{array}$ & $\begin{array}{l}9 \cdot 58 \\
(9 \cdot 61)\end{array}$ \\
\hline $5 f$ & $8-\mathrm{OCH}_{3}$ & $\mathrm{CH}_{3}$ & $\mathrm{CH}_{3}$ & $\begin{array}{l}\text { Ethanol + } \\
\text { dioxan }\end{array}$ & 260 & $68 \cdot 21$ & $\mathrm{C}_{28} \mathrm{H}_{23} \mathrm{~N}_{3} \mathrm{O}_{4}$ & $\begin{array}{c}72 \cdot 21 \\
(72 \cdot 25)\end{array}$ & $\begin{array}{c}4 \cdot 71 \\
(4 \cdot 94)\end{array}$ & $\begin{array}{l}9 \cdot 00 \\
(9 \cdot 03)\end{array}$ \\
\hline $5 \mathrm{~g}$ & 5,6-Benzo & $\mathrm{H}$ & $\mathrm{H}$ & $\begin{array}{l}\text { Ethanol + } \\
\text { dioxan }\end{array}$ & 265 & $76 \cdot 36$ & $\mathrm{C}_{29} \mathrm{H}_{23} \mathrm{~N}_{3} \mathrm{O}_{3}$ & $\begin{array}{c}75 \cdot 43 \\
(75 \cdot 48)\end{array}$ & $\begin{array}{l}4.95 \\
(4.98)\end{array}$ & $\begin{array}{l}9 \cdot 08 \\
(9 \cdot 11)\end{array}$ \\
\hline $5 \mathbf{h}$ & 5,6-Benzo & $\mathrm{CH}_{3}$ & $\mathrm{CH}_{3}$ & $\begin{array}{l}\text { Ethanol + } \\
\text { dioxan }\end{array}$ & 280 & $71 \cdot 61$ & $\mathrm{C}_{31} \mathrm{H}_{27} \mathrm{~N}_{3} \mathrm{O}_{3}$ & $\begin{array}{c}76 \cdot 01 \\
(76 \cdot 07)\end{array}$ & $\begin{array}{c}5 \cdot 49 \\
(5 \cdot 52)\end{array}$ & $\begin{array}{c}8.49 \\
(8 \cdot 58)\end{array}$ \\
\hline $5 i$ & $6-\mathrm{Cl}$ & $\mathrm{H}$ & $\mathrm{H}$ & Ethanol & 181 & $64 \cdot 00$ & $\mathrm{C}_{25} \mathrm{H}_{16} \mathrm{~N}_{3} \mathrm{O}_{3} \mathrm{Cl}$ & $\begin{array}{c}67 \cdot 96 \\
(68 \cdot 02)\end{array}$ & $\begin{array}{c}3 \cdot 58 \\
(3 \cdot 62)\end{array}$ & $\begin{array}{c}9 \cdot 48 \\
(9 \cdot 52)\end{array}$ \\
\hline $5 \mathbf{j}$ & $6-\mathrm{Cl}$ & $\mathrm{CH}_{3}$ & $\mathrm{CH}_{3}$ & Ethanol & 192 & $62 \cdot 20$ & $\mathrm{C}_{27} \mathrm{H}_{20} \mathrm{~N}_{3} \mathrm{O}_{3} \mathrm{Cl}$ & $\begin{array}{c}69 \cdot 03 \\
(69 \cdot 08)\end{array}$ & $\begin{array}{c}4 \cdot 21 \\
(4 \cdot 26)\end{array}$ & $\begin{array}{c}8.91 \\
(8.95)\end{array}$ \\
\hline $5 \mathbf{k}$ & $6-\mathrm{Br}$ & $\mathrm{H}$ & $\mathrm{H}$ & Ethanol & 198 & $63 \cdot 52$ & $\mathrm{C}_{25} \mathrm{H}_{16} \mathrm{~N}_{3} \mathrm{O}_{3} \mathrm{Br}$ & $\begin{array}{c}61.68 \\
(61.72)\end{array}$ & $\begin{array}{c}3 \cdot 26 \\
(3 \cdot 29)\end{array}$ & $\begin{array}{c}8.60 \\
(8.64)\end{array}$ \\
\hline 51 & $6-\mathrm{Br}$ & $\mathrm{CH}_{3}$ & $\mathrm{CH}_{3}$ & Ethanol & 206 & $62 \cdot 82$ & $\mathrm{C}_{27} \mathrm{H}_{20} \mathrm{~N}_{3} \mathrm{O}_{3} \mathrm{Br}$ & $\begin{array}{c}63 \cdot 48 \\
(63 \cdot 52)\end{array}$ & $\begin{array}{c}3 \cdot 09 \\
(3 \cdot 13)\end{array}$ & $\begin{array}{c}8 \cdot 19 \\
(8 \cdot 23)\end{array}$ \\
\hline
\end{tabular}

$(20 \mathrm{ml})$ was added $o$-phenylene diamine $(0.60 \mathrm{~g}$, 0.0055 mole) and $0.5 \mathrm{ml}$ of acetic acid. The reaction mixture was refluxed for $10 \mathrm{~h}$ and cooled. The separated solid was filtered and recrystallised from alcohol dioxane mixture to afford the benzodiazepene (5a) $(1.40 \mathrm{~g}, 70.88 \%)$ (table 6).

\section{References}

1. Richer A G and Sternbach L H 1968 Chem. Rev. 68747

2. Smith R H, Jorgen W L, Tirado R J and Lamb M L 1998 J. Med. Chem. 415272

3. Jadhav K P and Ingled D B 1983 Indian J. Chem. B22 180

4. Reddy R J, Ashok D and Sharma P N 1993 Indian J. Chem. B32 404
5. Satyanarayan K and Rao M N 1993 Indian J. Pharm. Sci. 55230

6. Dessarro G, Chimirri A, Dessaro A and Gitto R 1995 Eur. J. Med. Chem. 30925

7. Ishikawa S Jpn. Kokai Tokkyo Koho Jp. 61 96, 650; Chem. Abstr. $10611222 \mathrm{~g}$

8. Yoshihana I, Okuhara M and Yamamoto T Euri. Pat. Appl EP. 435 262; Chem. Abstr. 117 58904y

9. Knoevenagel E 1898 Berichte 31730

10. Bagchi P P and Ittyeah P I 1955 Agra Univ. J. Res. 145

11. Kovonagh F 1963 Analytical microbiology (New York: Academic Press) p. 125

12. Crawley J N and Godwin F K 1980 Preliminary of simple animal behavior model for effect of benzodiazepines. Pharm. Biochem. Behav. 167-170

13. Kilfoil T, Michel A and Montgomony D 1988 Psycho. Pharmacol. 28901 